\title{
Psychiatric Symptoms in Teachers from Danwon High School after Exposure to the Sinking of the Motor Vessel Sewol
}

\author{
Mi-Sun Lee ${ }^{1}$, Soo-Young Bhang ${ }^{2}$, Cheol-Soon Lee ${ }^{3}$, Hyoung Yoon Chang ${ }^{4,5}$, Ji-Youn Kim ${ }^{6}$, Ju-Hyun Lee, \\ Eunji Kim ${ }^{8}$, Seung-Min Bae ${ }^{9}$, Jang-Ho Park ${ }^{10}$, Hye-Jin Kim ${ }^{11}$, and Jun-Won Hwang ${ }^{11 凶}$ \\ 1'Department of Psychiatry, Eulji University Hospital, Seoul, Republic of Korea \\ ${ }^{2}$ Department of Psychiatry, Eulji University School of Medicine, Eulji University Hospital, Seoul, Republic of Korea \\ ${ }^{3}$ Department of Psychiatry, Gyeongsang National University School of Medicine, Gyeongsang National University Changwon Hospital, Changwon, \\ Republic of Korea \\ ${ }^{4}$ Department of Psychiatry, Ajou University School of Medicine, Suwon, Republic of Korea \\ ${ }^{5}$ Sunflower Center of Southern Gyeonggi for Women and Children Victims of Violence, Suwon, Republic of Korea \\ ${ }^{6}$ Goodmind Psychiatry Clinic, Suwon, Republic of Korea \\ ${ }^{7}$ Inarae Psychiatry Clinic, Seoul, Republic of Korea \\ ${ }^{8}$ Department of Psychiatry, Kyungpook National University Hospital, Daegu, Republic of Korea \\ ${ }^{9}$ Department of Psychiatry, Gachon University Gil Medical Center, Incheon, Republic of Korea \\ ${ }^{10}$ Department of Psychiatry, Ulsan University Hospital, University of Ulsan College of Medicine, Ulsan, Republic of Korea \\ ${ }^{11}$ Department of Psychiatry, Kangwon National University School of Medicine, Chuncheon, Korea
}

Objective The goal of this study was to describe the psychiatric symptoms in the teachers from Danwon High School who were exposed to the sinking of the Motor Vessel Sewol.

Methods Data were collected from 32 teachers who underwent psychiatric interventions by 16 volunteer psychiatrists for 3 months after the sinking of the Motor Vessel Sewol.

Results The most commonly diagnosed clinical diagnosis in the teachers were normal reaction, acute stress disorder and adjustment disorder. Psychiatric symptoms including anxiety, depressed mood and sleep disturbances were also observed.

Conclusion In the acute aftermath of the Sewol Ferry sinking on April 16, 2014, psychiatrists volunteered to provide professional psychiatric interventions to Danwon High School teachers. These results suggest the importance of crisis intervention focused on the teachers who are exposed to disasters. The implications for future research and interventions are discussed.

Psychiatry Investig 2017;14(6):900-903

Key Words Posttraumatic stress disorder, Trauma, Disaster psychiatry, Volunteer psychiatrists, Sinking of the Sewol Ferry.

\section{INTRODUCTION}

On April 16, 2014, the Motor Vessel Sewol capsized and sank 1.5 kilometers off Donggeochado, Jindo County, South Jeolla Province, on a voyage from Incheon to Jeju in South Korea. There were 476 people on the ferry: among which 325 students and 14 were teachers from Danwon High School in An-

Received: October 4, 2016 Revised: January 11, 2017

Accepted: January 29, 2017 Available online: August 25, 2017

$\triangle$ Correspondence: Jun-Won Hwang, MD, PhD

Department of Psychiatry, Kangwon National University Hospital, 156 Baengnyeong-ro, Chuncheon 24289, Republic of Korea

Tel: +82-33-258-9410, Fax: +82-33-254-1376, E-mail: huangjw@hanmail.net

(a) This is an Open Access article distributed under the terms of the Creative Commons Attribution Non-Commercial License (http://creativecommons.org/licenses/bync/4.0) which permits unrestricted non-commercial use, distribution, and reproduction in any medium, provided the original work is properly cited. san City. ${ }^{1}$ The disaster resulted a total of 304 deaths, including nine individuals whose bodies are not yet retrieved. Only 75 students and 3 teachers escaped before the ferry sank. ${ }^{2}$ Over 150 psychiatrists volunteered to provide crisis interventions to students and teachers of Danwon High School immediately after the disaster.

In general, disasters result in physical and mental health problems for both teachers and students. It also negatively affects the psychological well-being of the teachers. ${ }^{3}$ The psychological mental health of the teachers and their experiences with the disaster can significantly affect the school adjustment of the students and can lower the quality of classroom interactions. ${ }^{4}$ However, the majority of school-based interventions after disasters are only focused on the students. ${ }^{5-8}$

To the best of our knowledge, there are no reports regarding 
the psychiatric symptoms and clinical diagnoses among teachers after exposure to a disaster. Therefore, we report the psychiatric symptoms and clinical diagnoses of 32 teachers from Danwon High School.

\section{METHODS}

\section{Participants and procedures}

Data were obtained from a survey administered by the volunteer psychiatrists who conducted the crisis intervention sessions for the students and teachers of Danwon High School two years after the disaster. A total of 72 out of the 157 (45.9\%) volunteer psychiatrists answered the survey. Among them, 16 volunteer psychiatrists who conducted the interventions aimed at the teachers additionally performed a clinical interview to evaluate psychiatric symptoms in a total of 32 teachers. This study received approval from the Eulji University Institutional Review Board (IRB No. EMCS 2016-02-004).

\section{Demographics}

For the survey, the volunteer psychiatrists provided demographic data regarding their sex, age, average time per intervention per session (minutes), total time of intervention per day

Table 1. Demographic characteristics of the volunteer psychiatrists $(\mathrm{N}=16)$

\begin{tabular}{lc}
\hline \multicolumn{1}{c}{ Demographic parameters } & No. (\%) of subjects \\
\hline Age, mean (SD) & $41.00(6.0)$ \\
Sex & \\
$\quad$ Male & $3(18.7)$ \\
Female & $13(81.3)$ \\
Average time of intervention per & \\
1 session (minutes) & \\
$\leq 30$ & $3(18.7)$ \\
$30-40$ & $3(18.7)$ \\
$40-50$ & $6(37.6)$ \\
$\geq 50$ & $4(25.0)$ \\
Total time of intervention per 1 day (hours) & \\
$\leq 2$ & $14(87.5)$ \\
$2-3$ & $2(12.5)$ \\
Period of intervention & \\
Hyperacute stage & $10(62.5)$ \\
Acute stage & $5(31.3)$ \\
Subacute stage & $1(6.2)$ \\
The number of total intervention sessions & \\
1 & $11(68.8)$ \\
$2-3$ & $3(18.7)$ \\
$\geq 4$ & $2(12.5)$ \\
\hline
\end{tabular}

SD: standard deviation (hours), period of intervention, and the number of total intervention sessions. The basic demographic characteristics of the volunteer psychiatrists are shown in Table 1.

In addition, the volunteer psychiatrists reported the sex, age, chief complaints, observed psychiatric symptoms, and clinical diagnoses of the teachers. The chief complaints, psychiatric symptoms, and clinical diagnoses were available in multiple responses. These variables were classified based on a previous study. ${ }^{9}$ All analyses were conducted using Microsoft Office Excel version 2010 (Microsoft, Redmond, WA, USA).

\section{RESULTS}

The demographic characteristics, chief complaints, psychiatric symptoms and clinical diagnoses of the teachers are shown in Table 2. Among 32 teachers, 27 (84.4\%) of the teachers were women, and 15 (46.9\%) were in their thirties.

The majority of the reported chief complaints were related to their job performance. The most common chief complaint was difficulty performing work, which 20 teachers $(62.5 \%)$ answered positive. Difficulty in dealing with students was detected in 16 (50.0\%) of the teachers, and trouble with their colleagues was reported in 11 teachers (34.4\%). Additionally, 13 (40.6\%) teachers had a high risk of mental health, and 3 (9.4\%) teachers reported problems with their family members.

More than half of teachers reported anxiety $(\mathrm{n}=24,75.0 \%)$, depressed $\operatorname{mood}(\mathrm{n}=18,56.3 \%)$ and sleep disturbances $(\mathrm{n}=17$, $53.1 \%$ ). Other common symptoms included poor concentration ( $\mathrm{n}=13,40.6 \%$ ), hypervigilance/always being on guard for danger $(n=12,37.5 \%)$, palpitations ( $n=12,37.5 \%)$, being easily startled ( $\mathrm{n}=11,34.4 \%)$, and social isolation/withdrawal $(\mathrm{n}=11$, $34.4 \%$ ). In addition to the symptoms mentioned above, anger outbursts or aggressive behavior ( $\mathrm{n}=8,25.0 \%)$, loss of appetite $(\mathrm{n}=7,21.9 \%)$, recurrent distressing dreams/nightmares $(\mathrm{n}=6$, $18.8 \%$ ), headaches ( $\mathrm{n}=5,15.6 \%)$, flashbacks $(\mathrm{n}=4,12.5 \%)$, hyperventilation $(\mathrm{n}=1,3.1 \%)$, and hopelessness about the future $(\mathrm{n}=1,3.1 \%)$ were reported.

In 15 teachers (46.9\%), their difficulties were considered a normal reaction toward the disaster. However, 17 teachers (53.1\%) were clinically diagnosed in relation to the disaster: 7 (21.9\%) with acute stress disorder, 6 (18.8\%) with adjustment disorder, 2 (6.2\%) with major depressive disorder, 1 (3.1\%) with posttraumatic stress disorder (PTSD), and 1 (3.1\%) with anxiety disorder.

\section{DISCUSSION}

The sinking of the Motor Vessel Sewol resulted in widespread social and political reactions in South Korea.

Consequently, this study suggests that this disaster signifi- 
cantly contributed to the maintenance of psychiatric symptoms in teachers. In the first 3 months after the Sewol Ferry disaster, 16 volunteer psychiatrists provided professional services to 32 teachers, including a psychiatric assessment and crisis counseling.

Table 2. Demographic characteristics, chief complaints, psychiatric symptoms and clinical diagnoses of the teachers $(\mathrm{N}=32)$

\begin{tabular}{|c|c|}
\hline Variables & No. (\%) of details \\
\hline \multicolumn{2}{|l|}{ Sex } \\
\hline Male & $5(15.6)$ \\
\hline Female & $27(84.4)$ \\
\hline \multicolumn{2}{|l|}{ Age } \\
\hline $20-29$ & $11(34.4)$ \\
\hline $30-39$ & $15(46.9)$ \\
\hline $40-49$ & $6(18.7)$ \\
\hline \multicolumn{2}{|l|}{ Chief complaint* } \\
\hline Difficulty in performing work & $20(62.5)$ \\
\hline Difficulty about dealing with students & $16(50.0)$ \\
\hline High risk of mental health & $13(40.6)$ \\
\hline Difficulties for colleagues & $11(34.4)$ \\
\hline Problems with family & $3(9.4)$ \\
\hline \multicolumn{2}{|l|}{ Psychiatry symptoms* } \\
\hline Anxiety & $24(75.0)$ \\
\hline Depressed mood & $18(56.3)$ \\
\hline Sleep disturbances & $17(53.1)$ \\
\hline Problems with concentration & $13(40.6)$ \\
\hline $\begin{array}{l}\text { Hypervigilance/always being on guard } \\
\text { for danger }\end{array}$ & $12(37.5)$ \\
\hline Palpitation & $12(37.5)$ \\
\hline Being easily startled & $11(34.4)$ \\
\hline Social isolation/withdrawal & $11(34.4)$ \\
\hline Angry outbursts or aggressive behavior & $8(25.0)$ \\
\hline Loss of appetite & $7(21.9)$ \\
\hline Recurrent distressing dreams/nightmare & $6(18.8)$ \\
\hline Headache & $5(15.6)$ \\
\hline Flashback & $4(12.5)$ \\
\hline Hyperventilation & $1(3.1)$ \\
\hline Hopelessness about the future & $1(3.1)$ \\
\hline \multicolumn{2}{|l|}{ Clinical diagnosis* } \\
\hline Normal reaction & $15(46.9)$ \\
\hline Acute stress disorder & $7(21.9)$ \\
\hline Adjustment disorder & $6(18.8)$ \\
\hline Major depression disorder & $2(6.2)$ \\
\hline Posttraumatic stress disorder & $1(3.1)$ \\
\hline Anxiety disorder & $1(3.1)$ \\
\hline
\end{tabular}

*multiple response item; grade response percentages are based on the total sample
Teachers are frequently expected to support children psychologically in the aftermath of a disaster. ${ }^{10}$ In addition, they are in an important position to advocate for services and effective school-wide responses to crisis emergencies. However, they also experience psychological distress and psychiatric symptoms after a disaster. High levels of stress and mental health-related problems have been found in teachers within the school setting. ${ }^{11}$ Statistics outline that up to $30 \%$ of teachers are affected by burnout or psychological ill-being. ${ }^{12}$ Similar to these statistics and earlier studies, our data found that teachers had high levels of psychiatric symptoms. ${ }^{13}$ Relevant to the current study, the results of previous studies suggest that high emotional competence might act as a protective factor and reduce the effects of teacher work strain. ${ }^{14}$

In conclusion, crisis intervention by the volunteer psychiatrists meaningfully contributes to teacher's mental health following a disaster. These findings also suggest that comprehensive interventions are necessary for teachers after a disaster. Several limitations of the current study should be mentioned. First, the analyzed data were collected through the retrospective memory of the volunteer psychiatrists. Second, the sample was composed of only high school teachers, which limits the extent to which the findings can be generalized. However, these findings present an overview of teachers exposed to a disaster and emphasize the need for preventive strategies, including educational training plans for disasters, forming a teachers' support system, protecting teachers' safety and developing a national school disaster management plan. Furthermore, we need to have more organized training for emergency situations, disaster-related volunteer organizations and local government support, such as systematic intervention protocols, before working in an emergency site. ${ }^{15}$

Despite these limitations, the results of this study provide empirical evidence that crisis intervention to teachers exposed to a disaster is an important resource in mental health. In addition, our present results can be applied to provide appropriate support for the teachers in future large-scale disasters. Policy decisions should consider and support programs designed to enhance teachers' mental health when disaster occurs. ${ }^{16}$ Additionally, these results may suggest future treatment programs to improve mental health assistance for distressed teachers. Moreover, the data collected for this survey suggest a need for a teachers' support system and self-protection methods to prevent burnout.

\section{Acknowledgments}

We would like to give our heartfelt thanks to the volunteer psychiatrists who participated in this study.

This study was supported by a grant of the Korean Mental Health Technology R\&D Project, Ministry of Health \& Welfare, Republic of Korea (HM15C1058). 


\section{REFERENCES}

1. Kim J, Yoo CS. More than 300 people missing after South Korea ferry sinks-coastguard. Reuters April 20, 2014.

2. Woo H, Cho Y, Shim E, Lee K, Song G. Public Trauma after the Sewol Ferry Disaster: the role of social media in understanding the public mood. Int J Environ Res Public Health 2015;12:10974-10983.

3. Naghieh A, Montgomery P, Bonell CP, Thompson M, Aber JL. Organisational interventions for improving wellbeing and reducing work-related stress in teachers. Cochrane Database Syst Rev 2015;(4):CD010306.

4. Sandilos LE, Cycyk LM, Hammer CS, Sawyer BE, Lopez L, Blair C. Depression, control, and climate: an examination of factors impacting teaching quality in preschool classrooms. Early Educ Dev 2015;26: 1111-1127.

5. Gelkopf M, Berger R. A school-based, teacher-mediated prevention program (ERASE-Stress) for reducing terror-related traumatic reactions in Israeli youth: a quasi-randomized controlled trial. J Child Psychol Psychiatry 2009;50:962-971.

6. Wolmer L, Hamiel D, Barchas JD, Slone M, Laor N. Teacher-delivered resilience-focused intervention in schools with traumatized children following the second Lebanon War. J Trauma Stress 2011;24:309-316.

7. Wolmer L, Hamiel D, Laor N. Preventing children's posttraumatic stress after disaster with teacher-based intervention: a controlled study. J Am Acad Child Adolesc Psychiatry 2011;50:340-348, e1-2.

8. Wolmer L, Laor N, Yazgan Y. School reactivation programs after disaster: could teachers serve as clinical mediators? Child Adolesc Psychiatr
Clin N Am 2003;12:363-381.

9. Pandya A, Katz CL, Smith R, Ng AT, Tafoya M, Holmes A, et al. Services provided by volunteer psychiatrists after $9 / 11$ at the New York City family assistance center: September 12-November 20, 2001. J Psychiatr Pract 2010;16:193-199.

10. Pfefferbaum RL, Fairbrother G, Brandt EN Jr, Robertson MJ, Gurwitch $\mathrm{RH}$, Stuber J, et al. Teachers in the aftermath of terrorism: a case study of one New York City school. Fam Community Health 2004;27:250259.

11. Sass DA, Seal AK, Martin NK. Predicting teacher retention using stress and support variables. J Educ Admin 2011;49:200-215.

12. Milatz A, Luftenegger M, Schober B. Teachers' relationship closeness with students as a resource for teacher wellbeing: a response surface analytical approach. Front Psychol 2015;6:1949.

13. Maslach C. What have we learned about burnout and health? Psychol Health 2001;16:607-611.

14. Brackett M, Palomera R, Mojsa-Kaja J, Regina M, Salovey P. Emotionregulation ability, burnout, and job satisfaction among british secondary-school teachers. Psychol Sch 2010;47:406-417.

15. Bills CB, Levy NA, Sharma V, Charney DS, Herbert R, Moline J, et al. Mental health of workers and volunteers responding to events of 9/11: review of the literature. Mt Sinai J Med 2008;75:115-127.

16. Flook L, Goldberg SB, Pinger L, Bonus K, Davidson RJ. Mindfulness for teachers: a pilot study to assess effects on stress, burnout and teaching efficacy. Mind Brain Educ 2013;7. 